\title{
Case Report: Novel Coronavirus-A Potential Cause of Acute Pancreatitis?
}

\author{
Syed Muhammad Mashhood Ali Bokhari and Fatima Mahmood \\ Nishtar Medical College and Hospital, Multan, Pakistan
}

\begin{abstract}
A 32-year-old medical practitioner presented to the emergency department with complaints of severe abdominal pain, fever, and vomiting, 1 week after the diagnosis of COVID-19. The patient did not report any comorbid conditions, alcohol usage, or gallstone disease. Laboratory and radiological investigations revealed the diagnosis of acute pancreatitis. He underwent conservative management and was discharged after 3 days of hospital admission. This case highlights a possible association between COVID-19 and acute pancreatitis, and the need for clinicians to carefully evaluate patients presenting with gastrointestinal complaints during the current pandemic.
\end{abstract}

\section{CASE DESCRIPTION}

A 32-year-old male patient presented to the outpatient clinic at Nishtar Hospital, Multan, Pakistan, with a history of relapsing fever, sore throat, productive cough, myalgia, and diarrhea for 1 week. The patient was a medical physician currently working in the medicine ward. He did not report any prior comorbidities or high-risk travel history.

Considering the exposure history, a reverse transcriptasePCR for COVID-19 was obtained. Initially, the patient's temperature was $38^{\circ} \mathrm{C}$, the blood pressure $110 / 80 \mathrm{mmHg}$, the pulse rate 110 beats per minute, the respiratory rate 16 breaths per minutes, and oxygen saturation 99-100\% while breathing in ambient air. He was managed with simple supportive measures.

After 21 hours, his tests for COVID-19 were reported positive. The patient chose to self-isolate at home until his symptoms subsided and his results came negative. He was advised to report to the emergency department in case of severe dyspnea, chest pain, cyanosis, or altered mental status.

A week after the diagnosis, the patient reported to the emergency department with severe mid-epigastric pain radiating to the back accompanied by intermittent high fevers, chills, and non-biliary vomiting. He was noted to have a white cell count of $12 \times 10^{9} / \mathrm{L}$ and glucose levels of $192 \mathrm{mg} / \mathrm{dL}$. Liver function tests revealed aspartate aminotransferase of $29 \mathrm{IU} / \mathrm{L}$ and alanine aminotransferase of $27 \mathrm{IU} / \mathrm{L}$. Kidney functions were normal, serum triglycerides were $150 \mathrm{mg} / \mathrm{dL}$, and serum calcium was $8.9 \mathrm{mg} / \mathrm{dL}$. Serum amylase was $672 \mathrm{IU} / \mathrm{L}$ and serum lipase $721 \mathrm{IU} / \mathrm{L}$ (Table 1). The patient reported no history of use of alcohol.

Ultrasound in the emergency ward showed no signs of inflammation of the gall bladder or cholelithiasis. Computed axial tomography scan of the abdomen revealed a bulky and swollen pancreas with significant peripancreatic inflammatory changes and fluid collection along the gastrosplenic ligament. Otherwise, the pancreas was non-enhancing, with no evidence of low-attenuation areas in the pancreatic parenchyma to suggest necrosis (Figure 1).

Considering a diagnosis of acute pancreatitis, the patient was kept nothing per oral and on intravenous fluids, analgesics, antibiotics, and antiemetics. The patient's intake and

*Address correspondence to Syed Muhammad Mashhood Ali Bokhari, Department of Surgery, Nishtar Medical College and Hospital, Nishtar Rd., Multan, Pakistan. E-mail: mashhood.bokhari@ hotmail.com output were monitored, and he was gradually mobilized from bed. Alongside, incentive spirometry and chest physiotherapy were initiated.

Over the next 3 days, his lipase was constantly monitored and showed a downward trend, 380, 198, and $56 \mathrm{IU} / \mathrm{L}$. On day 3 , his symptoms improved, and he was started on clear fluids and soft diet through oral route, which was successfully tolerated. The patient was discharged home without any sequelae.

\section{DISCUSSION}

In early December 2019, Wuhan City, China, experienced a sudden outbreak of pneumonia. Investigation led to isolation of a novel coronavirus as the cause of outbreak. The WHO named it as COVID-19 on February 11, 2020. This virulent pathogen rapidly spread in China and other regions in the world. Till date, the virus continues to cause a devastating pandemic worldwide, threatening global health and affecting approximately $4,000,000$ people. It causes great threats to the growth of economy and society. ${ }^{1,2}$

Respiratory symptoms such as cough, shortness of breath, and sore throat appear to be the pathognomonic signs in a patient infected with the coronavirus; however, the literature

TABLE 1

Clinical laboratory results

\begin{tabular}{lccc}
\hline \multicolumn{1}{c}{ Variable } & Reference range & Day 1 & Day 2 \\
\hline C-reactive protein (mg/dL) & $0-0.5$ & 1.58 & - \\
Lipase (U/L) & $6-51$ & 721 & 380 \\
Amylase (U/L) & $28-100$ & 672 & - \\
Glucose (mg/dL) & $80-160$ & 192 & 153 \\
Blood urea nitrogen (mg/dL) & $6-20$ & 11 & - \\
Creatinine (mg/dL) & $0.9-1.3$ & 1.3 & 1.1 \\
Sodium (mmol/L) & $136-145$ & 136 & 140 \\
Potassium (mmol/L) & $3.5-5.1$ & 3.7 & 4.2 \\
Chloride (mmol/L) & $98-107$ & 101 & 107 \\
Calcium (mg/dL) & $8.6-10.2$ & 8.9 & - \\
Bicarbonate (mmol/L) & $20-31$ & 20.4 & 26.4 \\
Lactate dehydrogenase (U/L) & $120-246$ & 212 & - \\
Aspartate aminotransferase (U/L) & $10-40$ & 29 & - \\
Alanine aminotransferase (U/L) & $10-49$ & 27 & - \\
Alkaline phosphatase (U/L) & $46-116$ & 82 & - \\
Total bilirubin (mg/dL) & $0.1-1.2$ & 1.0 & - \\
Total cholesterol & & & \\
Triglyceride & $13.7-16.3$ & 14.2 & - \\
Hemoglobin (g/dL) & $41.9-48.7$ & 42.8 & - \\
Hematocrit (\%) & $4.0-10.0$ & 12 & 11.2 \\
White blood count $\left(\times 10^{9} / \mathrm{L}\right)$ & $150-400$ & 247 & - \\
Platelets $\left(\times 10^{9} / \mathrm{L}\right)$ & & & \\
\hline
\end{tabular}




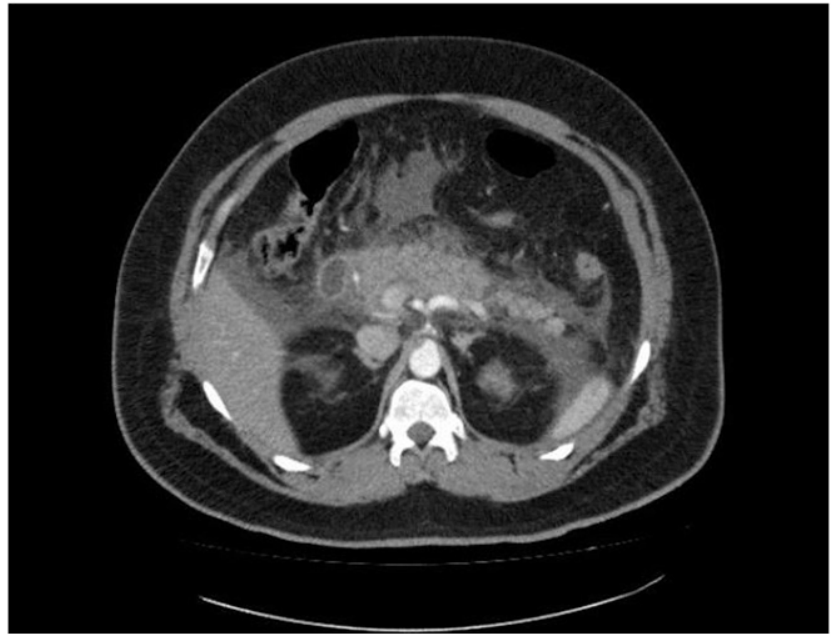

FIGURE 1. CT scan showing a swollen pancreas with inflammatory changes in surrounding areas.

regarding its extrapulmonary symptoms are still evolving. With the increase in the number of cases and accumulation of data on possible signs and symptoms, it appears that gastrointestinal symptoms are fairly common in COVID-19 patients. A comprehensive study conducted in Hubei, China, evaluated 204 patients who tested positive for COVID-19, of whom $50.5 \%$ reported some gastrointestinal disturbance such as diarrhea, anorexia, vomiting, or abdominal pain. A rare group of patients presented with gastrointestinal symptoms only without any respiratory symptoms. ${ }^{3}$

In our case, the diagnosis of pancreatitis in this patient appears to be idiopathic in the absence of comorbid conditions, cholelithiasis, alcohol usage, trauma, or recent invasive procedures such as endoscopic retrograde cholangiopancreatography. However, around $10 \%$ of cases are due to miscellaneous factors such as viral, bacterial, or parasitic infections. Notably, Coxsackievirus, herpes simplex virus, mumps, human immunodeficiency virus, and Mycoplasma, among several others, are responsible for causing infectious pancreatitis. ${ }^{4}$ Similar cases have been reported recently from Newport (United Kingdom) and Denmark, where multiple patients with COVID-19 disease presented with complaints of acute pancreatitis. ${ }^{5,6} \mathrm{~A}$ study in Wuhan city showed the prevalence of pancreatic injury in nine of 52 patients admitted in a hospital. It suggested that the pancreatic injury could be due to heavy expression of angiotensin converting enzyme or harmful systemic immune response induced by COVID-19 infection. ${ }^{7}$ Based on this case and the aforementioned studies, we recommend further studies to be conducted to evaluate any possible association between COVID-19 and acute pancreatitis.

\section{CONCLUSION}

This is a case describing the incidence of pancreatitis in a COVID-19-positive patient. We encourage medical practitioners to carefully evaluate gastrointestinal symptoms and measure serum amylase and lipase levels in patients presenting with abdominal pain and COVID-19.

Received June 1, 2020. Accepted for publication July 1, 2020.

Published online July 8, 2020.

Acknowledgment: Publication charges for this article were waived due to the ongoing pandemic of COVID-19.

Authors' addresses: Syed Muhammad Saud Ali Bokhari, Shifa College of Medicine, Islamabad, Pakistan, E-mail: m.saudbukhari@gmail.com. Fatima Mahmood, Nishtar Medical College and Hospital, Multan, Pakistan, E-mail: fatimamahmood027@gmail.com.

This is an open-access article distributed under the terms of the Creative Commons Attribution (CC-BY) License, which permits unrestricted use, distribution, and reproduction in any medium, provided the original author and source are credited.

\section{REFERENCES}

1. Phelan AL, Katz R, Gostin LO, 2020. The novel coronavirus originating in Wuhan, China: challenges for global health governance. JAMA 323: 709-710.

2. Wang MP, Anderson N, Yang E, Poon L, Charlton C, Zelyas N, Persing D, Rhoads D, Babcock H, 2020. The SARS-CoV-2 outbreak: diagnosis, infection prevention, and public perception moderators. Clin Chem, doi: 10.1093/clinchem/hvaa080.

3. Pan $L$ et al., 2020. Clinical characteristics of COVID-19 patients with digestive symptoms in Hubei, China. Am J Gastroenterol 115: 766-773.

4. Rawla P, Bandaru SS, Vellipuram AR, 2017. Review of infectious etiology of acute pancreatitis. Gastroenterol Res 10: 153-158.

5. Anand ER, Major C, Pickering O, Nelson M, 2020. Acute pancreatitis in a COVID-19 patient. Br J Surg 107: e182.

6. Hadi A, Werge M, Kristiansen KT, Pedersen UG, Karstensen JG, Novovic S, Gluud LL, 2020. Coronavirus Disease-19 (COVID19) associated with severe acute pancreatitis: case report on three family members. Pancreatology 20: 665-667.

7. Wang F, Wang H, Fan J, Zhang Y, Wang H, Zhao Q, 2020. Pancreatic injury patterns in patients with COVID-19 pneumonia. Gastroenterology, doi: 10.1053/j.gastro.2020.03.055. 